\title{
Parametric Heat Transfer Study of Bolted Joints
}

\author{
M. B. H. Mantelli* \\ Federal University of Santa Catarina, Florianópolis, SC 88037-001, Brazil \\ and \\ M. M. Yovanovich† \\ University of Waterloo, Waterloo, Ontario N2L 3E5, Canada
}

\begin{abstract}
A parametric study is performed to eliminate some of the thermal resistances of the complex network obtained for bolted joints. The analytical models for material, contact, and radiation resistances, developed by the authors and available in the literature, are used. Thermal resistances and resistance paths are compared among themselves. The resistances found to be unimportant are excluded from the circuit and a simplified thermal circuit is obtained. A sensitivity analysis of the parameters used in the formulation of the overall thermal resistance of the simplified network is performed. This study points out the important parameters, which should be precisely determined. The comparison of the present model with the experimental data and with the literature correlations shows the accuracy of the simplified thermal circuit. Except for special cases, large variations of the parameters that affect the contact resistance do not significantly influence the overall thermal resistance of the bolted joint. Therefore, the precise determination of the contact conductance may not be crucial for many bolted joint configurations used in practical applications.
\end{abstract}

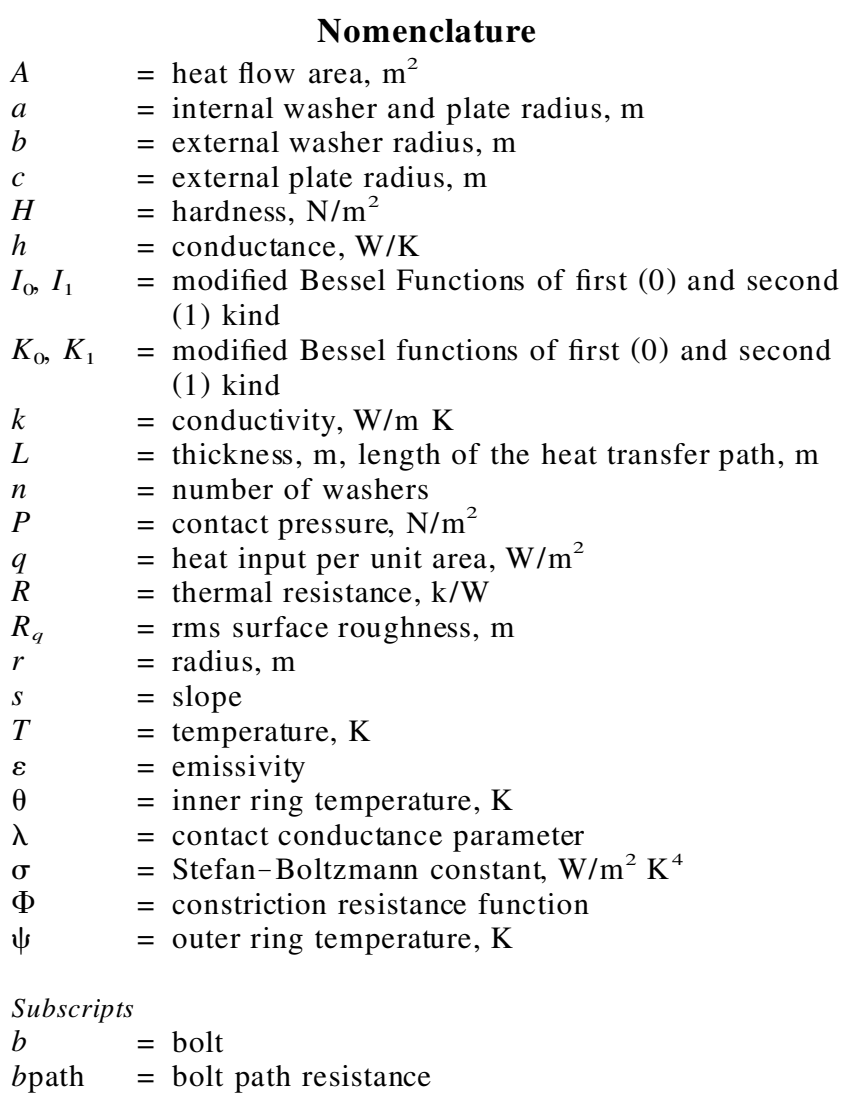

Presented as Paper 97-2459 at the AIAA 32nd Thermophysics Conference, Atlanta, GA, June 23-25, 1997; received Aug. 18, 1997; revision received March 6, 1998; accepted for publication March 10 1998. Copyright (C) 1998 by M. B. H. Mantelli and M. M. Yovanovich. Published by the American Institute of Aeronautics and Astronautics, Inc., with permission.

* Senior Development Engineer and Invited Researcher from National Space Research Institute, São José dos Campos, Brazil. Member AIAA.

$†$ Professor and Director, Mechanical Engineering Department. Fellow AIAA.

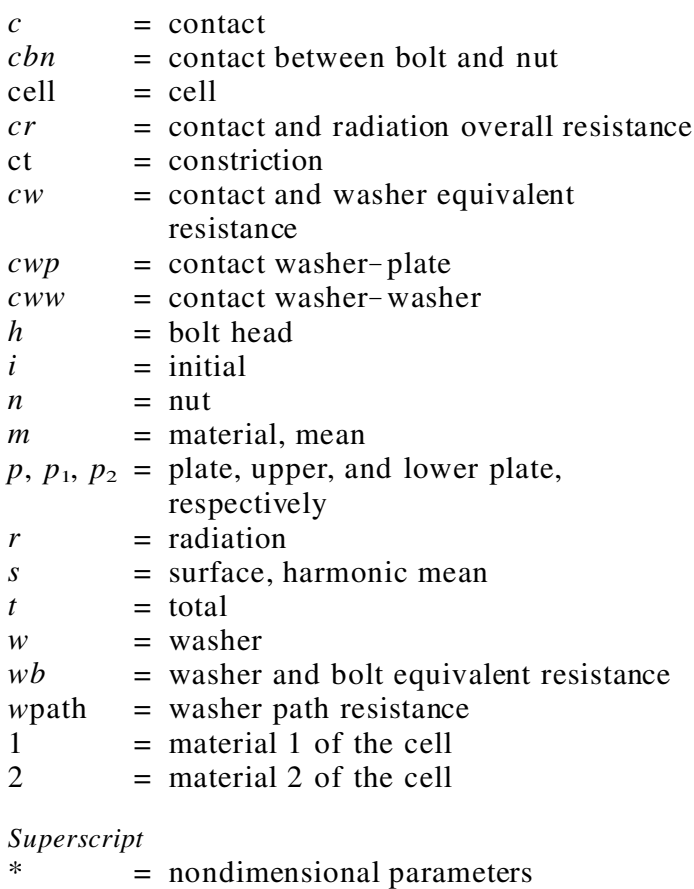

\section{Introduction}

A BOLTED joint is defined as the junction formed by two plates connected by a bolt, a nut, and some washers. Bolted joints are used in numerous applications, varying from domestic appliances to spacecrafts. From the thermal point of view, they are important when they affect the thermal performance of the system. This is the case of many electronic equipment, such as the computers, where printed circuit boards are connected to their structural frames by bolted joints. This is also the case of solar water-heating systems, where bolted joints are used to connect tubes to solar collector panels. Bread bakery ovens may be another application, because bolted joints can be used to fasten thermosyphons to baking trays. Satellite applications are the motivation of the present work.

At the space environment, in the vacuum, convection heat transfer is negligible. For the inside compartments of satellites, 
which do not present large temperature differences and, therefore, low thermal radiation exchange, the conduction is the principal mode of heat transfer. Boxes that contain electronic equipment are connected to the structural panels by bolted joints. Sometimes these junctions are the only way through which heat is transferred from the inside to the outside environment of the satellite.

In the current literature, the determination of the thermal resistances of bolted joints is concentrated mostly in the characterization of the contact resistance between plates fastened by a bolt. ${ }^{1-5}$ Less often, the constriction resistance of plates, subjected to specific boundary conditions, is also studied., Some heat transfer paths, such as the bolt path, have simply been neglected.

A simple compact model to predict the overall thermal resistance of bolted joints was developed. ${ }^{8,9}$ The first step in the modeling was to identify all of the thermal resistances found in a bolted joint, which were grouped in a network. The circuit that was obtained was rather complicated. A comparative study to eliminate some of these resistances from the circuit was performed. This study is presented in this paper.

The present paper is divided into two major sections. First, a comparative study of the resistances is conducted. As a result, many resistances and resistance paths are eliminated and a simplified thermal circuit is obtained. The conditions, which permit the elimination of these resistances, are also established. Second, a sensitivity analysis of the parameters used in the model applied to the simplified circuit (simplified model) is presented. The objective of this analysis is to point out the controlling parameters for the overall thermal resistance, which should be obtained with accuracy. Finally, a comparison of the model with data and other models is shown. Interesting results, with many implications in real applications, are presented.

\section{Bolted Joint Configuration}

This present study is based on the SCD1 (Brazilian Data Collection Satellite) bolted joints, which are considered typical for space applications. The SCD1 mounting is composed of two thin plates, of similar thicknesses and the same materials, connected by a bolt, as shown in Fig. 1. Identical washers are positioned between the plates, between the bolt head and the upper plate and between the nut and the lower plate. The dimensions of the mounting, thermophysical properties, contact pressure, etc., are shown next. $a=0.0037 \mathrm{~m}, b=0.0083 \mathrm{~m}$, $c=0.0889 \mathrm{~m}, n=3, r_{n}=0.0025, L_{w}=0.0032 \mathrm{~m}, L_{p}=0.0064$ $\mathrm{m}, L_{n}=0.0032 \mathrm{~m}, r_{b}=0.002 \mathrm{~m}, k_{w}=14.8 \mathrm{~W} / \mathrm{m}{ }^{\circ} \mathrm{C}, k_{p}=210$ $\mathrm{W} / \mathrm{m}{ }^{\circ} \mathrm{C}, k_{b}, k_{n}=14.8 \mathrm{~W} / \mathrm{m}{ }^{\circ} \mathrm{C}, P=10 \mathrm{MPa}, H_{p}=1063 \mathrm{MPa}$, $H_{w}=6517 \mathrm{MPa}, \varepsilon_{p}=0.032, \varepsilon_{w}=0.032, R_{q} / s_{c w w}=3.55710^{-6}$ $\mathrm{m}, R_{q} / s_{\text {cwp }}=7.63910^{-6} \mathrm{~m}$, and $T=300 \mathrm{~K}$. The range of variation of the parameters used in this study is based on the presented values. A vacuum environment is assumed; therefore, convection heat transfer is considered negligible.

\section{Parametric Study}

The analogy between electrical and thermal circuits is used for the determination of the overall thermal resistance of bolted joints. After the identification of all the thermal resistances, they are connected in a network, as presented in the left side of Fig. 2. This circuit is rather complicated. A parametric study

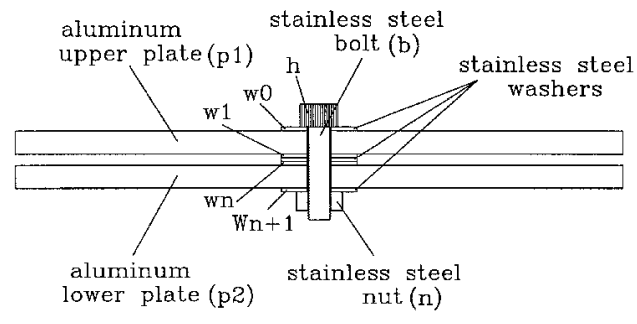

Fig. 1 Typical satellite bolted joint. is performed with the objective of simplifying the network by the elimination of some resistances with negligible error. Mantelli and Yovanovich ${ }^{9}$ models are used in the formulation of the resistances.

Four steps are adopted in this parametric study. In the first step, to understand the basic heat transfer mechanisms, a cell resistance is defined. A cell resistance is useful for the study of the relative importance of the material, contact, and radiation resistances, because it is insulated from the influences of other resistances of the network. The second step is the comparison of the total resistances of the two paths in parallel: The washer and plate path and the bolt path (see Fig. 2). These resistance paths are composed of several material and contact resistances. In the third step, to simplify the mathematical model, the terms of the formulation used in the constriction resistance model are compared among themselves. Finally, in the fourth step, the total resistance resulting from these two paths in parallel is compared with the radiation resistance between the plates. These steps are presented in detail in the following sections. Nondimensional parameters are used throughout this study, to make the results general.

\section{Analysis of the Cell Resistance}

In this section, the basic problem of two solids in physical contact is investigated. The objective of this analysis is to study the relative importance of an elemental interface problem. The same elemental cell may appear several times in a bolted joint. In this study, the contacts between the bolt head and the upper washer and between the nut and the lower washer form similar cells. In the same way, the two contacts between washers form similar cells (see Fig. 1).

A cell is defined as any two bodies, 1 and 2 , of $L_{1}$ and $L_{2}$ thicknesses, $k_{1}$ and $k_{2}$ conductivities, and at $T_{1}$ and $T_{2}$ temperatures, which are in contact over one of their surfaces. They are subject to a heat flux, which is supplied to the external face of one of the bodies and removed from the external face of the other body. The cell resistance circuit is composed of the material resistance of the first plate $R_{m 1}$, the resistance between the contacting surfaces, (formed by the contact $R_{c}$ and radiation $R_{r}$ resistances connected in parallel) and of the material resistance of the lower plate $R_{m 2}$. Figure 3 shows the cell resistance of an interface between two washers and the associated thermal circuit. In this work, the material resistance is common for two different cells and, therefore, half of the material resistance is considered part of one cell and half part of the other. The material resistances are combined into one resistance, $R_{m}$ (see second network, Fig. 3) and its expression is ${ }^{9}$

$$
R_{m}=\frac{1}{2} \frac{\left(L_{1} k_{2}+L_{2} k_{1}\right)}{k_{1} k_{2} \pi\left(b^{2}-a^{2}\right)}
$$

Several contact resistance models are found in the literature. A study was performed in Mantelli et al. ${ }^{10}$ to point out which one is the best for the present case. This study showed that most of the available correlations provide reasonable results. The contact resistance correlation of Yovanovich, ${ }^{11}$ based on the Cooper et al. ${ }^{12}$ plastic deformation theory was selected

$$
R_{c}=\frac{R_{q} / s}{1.25 k_{s}(P / H)^{0.95} \pi\left(b^{2}-a^{2}\right)}
$$

where $H$ is the microhardness of the softer contact material, and $R_{q} / s$ is the ratio between the rms surface roughness and its average slope.

The radiation resistance between the contacting surfaces is calculated based on the assumption that the heat is exchanged between two infinite parallel grey plates. The resulting expression is

$$
R_{r}=\frac{1 / \varepsilon_{1}+1 / \varepsilon_{2}-1}{4 \sigma T_{m}^{3} \pi\left(b^{2}-a^{2}\right)}
$$




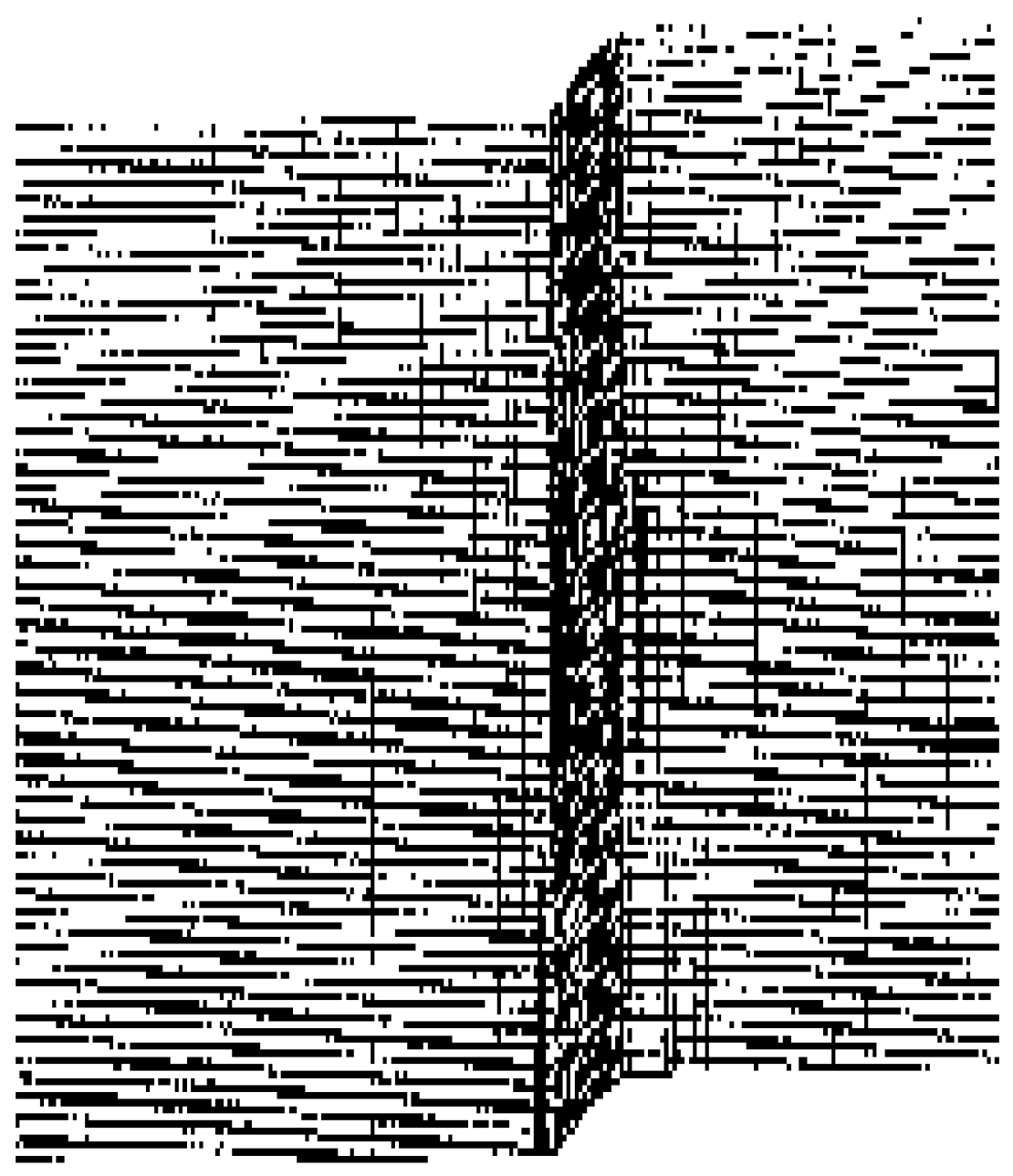

Fig. 2 Complete and simplified thermal circuits.

where $\varepsilon_{1}$ and $\varepsilon_{2}$ are the emissivities of the contacting surfaces of materials 1 and 2, respectively. The following nondimensional parameters are used:

$$
\begin{gathered}
a^{*}=\frac{a}{b}, \quad L_{s}^{*}=\frac{L_{s}}{b}, \quad T_{m}^{*}=\frac{T_{m} k_{s} L_{s}}{q b^{2}} \\
h_{m}^{*}=\frac{b\left(k_{1}+k_{2}\right)}{L_{1} k_{2}+L_{2} k_{1}}, \quad h_{c}^{*}=\frac{1.25(P / H)^{0.95} b}{R_{q} / s} \\
h_{r}^{*}=\frac{4 \sigma\left(T_{m}^{*}\right)^{3} b}{k_{s}\left(1 / \varepsilon_{1}+1 / \varepsilon_{2}-1\right)}, \quad R^{*}=R k_{s} L_{s} \\
k_{s}=2 \frac{k_{1} k_{2}}{k_{1}+k_{2}}, \quad L_{s}=2 \frac{L_{1} L_{2}}{L_{1}+L_{2}}
\end{gathered}
$$

The $h^{*}$ groups represent nondimensional conductances. $k_{s}$ and $L_{s}$ are the harmonic means of the conductivities and lengths of materials 1 and 2, respectively. Their expressions are used in the nondimensional form of other contact parameters, after the appropriate substitution of $k_{1}, k_{2}, L_{1}$, and $L_{2}$. Other nondimensional temperatures are obtained through the $T_{m}^{*}$ expression with the substitution of $T_{m}$ by the appropriate temperature.

The nondimensional form of the material, contact, and radiation resistances are, respectively

$$
R_{m}^{*}=\frac{L_{s}^{*}}{h_{m}^{*} \pi\left(1-a^{*^{2}}\right)}, \quad R_{c}^{*}=\frac{L_{s}^{*}}{h_{c}^{*} \pi\left(1-a^{*^{2}}\right)}, \quad R_{r}^{*}=\frac{L_{s}^{*}}{h_{r}^{*} \pi\left(1-a^{*^{2}}\right)}
$$

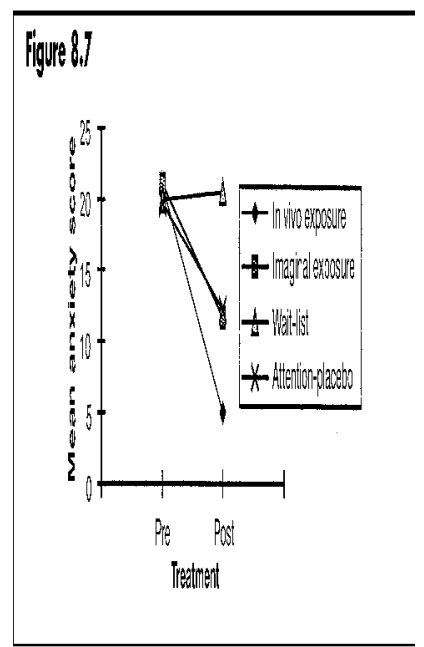

The overall nondimensional thermal resistance of the cell is

$$
R_{\text {cell }}^{*}=R_{m}^{*}+\left(\frac{1}{R_{c}^{*}}+\frac{1}{R_{r}^{*}}\right)^{-1}
$$

Figure 4 presents a parametric plot of the resistance $R_{c r}^{*}$, obtained by the combination in parallel of the contact $R_{c}^{*}$ and radiation $R_{r}^{*}$ resistances, as a function of $R_{c}^{*}$, for various values of $R_{r}^{*}$. The ranges of variation of the resistances $R_{c}^{*}$ and $R_{r}^{*}$ are based on their nominal values, obtained from the data pre- 


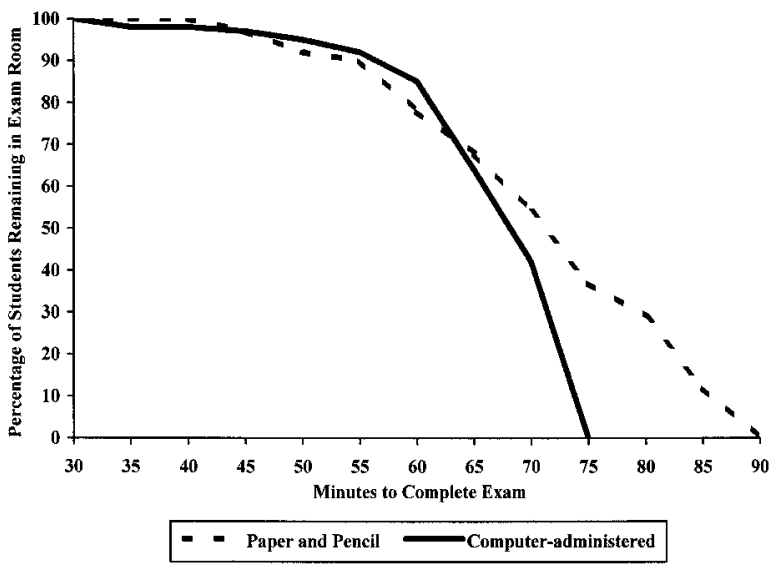

Fig. $4 R_{\sigma}^{*}$ as a function of $R_{c}^{*}$ for $R_{r}^{*}$ varying from 20.5 to 205.0 .

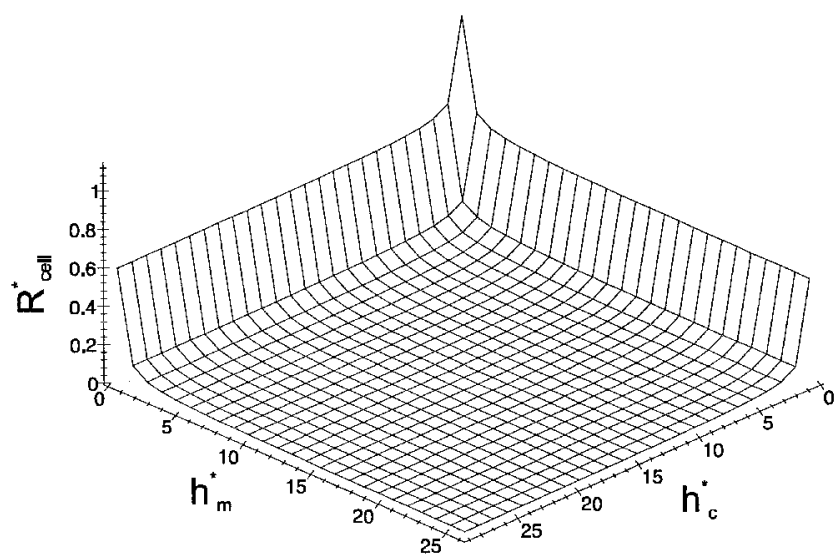

Fig. $5 R_{\text {cell }}^{*}$ as a function of $h_{m}^{*}$ and $h_{c}^{*}$.

sented earlier. Their limits are one order of magnitude lower and one order of magnitude higher than the nominal resistances. Therefore, the position of the present case in the plot is close to the origin. This same criterion is also used in the other plots.

From Fig. 4, one observes that $R_{c r}^{*}$ varies almost linearly with $R_{c}^{*}$. The lower bound of $R_{r}^{*}$ is 20.5 , which is a much larger value than the upper limit of $R_{c}^{*}$. This means that $R_{r}^{*}$ can be eliminated from the circuit. The thermal circuit on the bottom right side of Fig. 3 shows the cell circuit without the radiation resistance. As a conclusion, all of the radiation resistances presented in Fig. 2, which are parallel to the contact resistances, are eliminated from the network. The simplified cell resistance expression is

$$
R_{\text {cell }}^{*}=R_{m}^{*}+R_{c}^{*}
$$

The relative importance of the contact and material conductances in the cell resistance $R_{\text {cell }}^{*}$ is observed in Fig. 5. Obviously, the influences of both conductances are very similar. Therefore, for the cell resistance, both the material and contact conductances are important. From this figure, one observes that the cell resistance is very insensitive to changes in the conductances when they present values greater than 3 . On the other hand, when these conductances assume values less than 2 , the cell resistance starts to increase quickly. Mantelli ${ }^{8}$ adopted a value of 2.3 as the transition point. Actually, the contact conductance is much more susceptible to variations than the material conductance, because it depends on more parameters [see Eqs. (1) and (2)].

It is important to observe that the analysis developed in this section is general and valid for any two materials in contact. Figures 4 and 5 can be used to estimate the resistances for the cases where parameters are within the range of variation used to generate the surfaces, particularly when $h_{c}^{*}$ and $h_{m}^{*}$ are greater than 3 .

\section{Analysis of the Washer and Bolt Heat Transfer Paths}

The objective of this study is to compare the washer and bolt heat transfer paths that are parallel to each other. The analysis developed in this section is not as general as the cell resistance analysis, because some simplifications must be imposed on the physical model. A bolted joint has several different components that are subjected to different temperature levels. An exact solution would require each of the resistances shown in Fig. 2 to be calculated individually, at its own temperature. In this case, because the network is symmetric, the temperatures of all resistances are considered the same, equal to the average temperature of the mounting.

Using the idea of cell resistance, it is possible to make several simplifications to the resistance network shown in Fig. 2. The cell resistances between similar materials and presenting similar geometries are assumed equals. One should note that the contact resistances between washer $w_{1}$ and upper plate $p_{1}$ are included in the formulation of the plate constriction resistance. ${ }^{9}$ The same can be observed for the lower plate $p_{2}$.

Therefore, the washer and plate-path thermal resistance is given by

$$
R_{\text {wpath }}=R_{m w 1}+(n-1) R_{w w}
$$

where $R_{w w}$ is the cell resistance between washers, $R_{m w 1}$ is the material resistance of washer $w_{1}$, and $n$ is the number of washers between plates. The material resistance is determined through a unidimensional expression $(R=L / k A)$.

Similarly, the bolt path thermal resistance is given by

$$
R_{b \text { path }}=2 R_{\text {un }}+R_{c b n}+R_{m b}
$$

where $R_{m b}$ is the material resistance of the bolt shaft, $R_{w n}$ is the cell resistance between the washer and nut, and $R_{c b n}$ is the contact resistance between the bolt and nut. The bolt head and nut temperature distributions are bidimensional, but, in this work, they are considered unidimensional. This simplification is counterbalanced increasing the length of the bolt shaft $L_{b}$, by two times $L_{n} / 2$ and two times $r_{n} / 2 . L_{n} / 2$ is half the thicknes of the bolt head and the nut (this accounts for the resistances in the axial direction), and $r_{n} / 2$ is half the radius of the nut and the bolt head (this accounts for the resistances in the radial direction). The bolt head and nut dimensions are considered the same. Therefore, the heat transfer path length in the bolt shaft is given by the expression

$$
L=L_{b}+2\left(L_{n} / 2\right)+2\left(r_{n} / 2\right)
$$

The contact area used to calculate the bolt and nut contact resistance is assumed to be equal to the nut cylindrical projection area on the bolt shaft $\left(A_{c}=2 \pi r_{b} L_{n}\right)$. The equivalent nondimensional washer-bolt path thermal resistance is

$$
R_{w b}^{*}=\left(\frac{1}{R_{w \text { path }}^{*}}+\frac{1}{R_{b \text { path }}^{*}}\right)^{-1}
$$

In Fig. 6, a plot of the nondimensional resistances $R_{w b}^{*}$ as a function of $R_{w p a t h}^{*}$ for several values of $R_{b \text { path }}^{*}$ is presented. In this case, the ranges of variation of $R_{w \text { path }}^{*}$ and of $R_{b \text { path }}^{*}$ are obtained by taking actual limits for two of the most important variables: thickness of the washer and conductivity of the bolt. This procedure is adopted to keep the resistances within realistic values. From this plot one notes that most of the curves collapse into an almost linear curve with a slope close to one. Also, one observes that it is hard to distinguish different $R_{\text {bpath }}^{*}$ curves. Therefore, one can conclude that the $R_{b \text { path }}^{*}$ resis- 


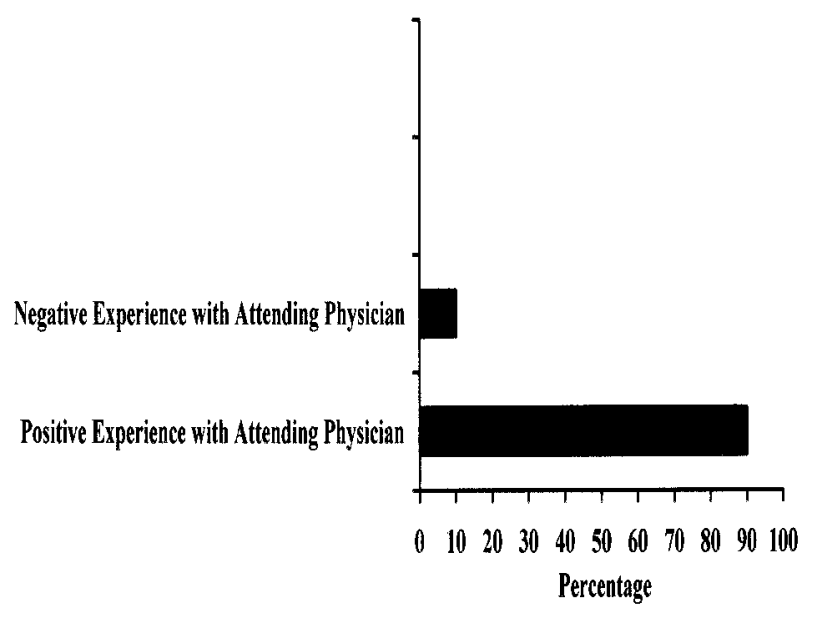

Fig. $6 R_{u b}^{*}$ as a function of $R_{w p a t h}^{*}$ for several values of $R_{b \text { pattr }}^{*}$

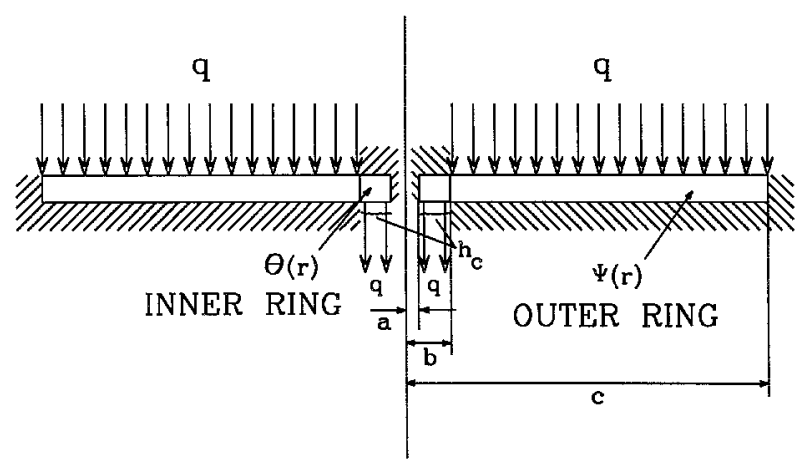

Fig. 7 Physical model of the constriction resistance.

tance can be removed from the network shown in Fig. 2. However, other plots (not presented here) show that rare situations may happen where the $R_{b \text { path }}^{*}$ may not be negligible. One example is the case when the area of the bolt section is approximately the same area of the washer.

\section{Analysis of the Constriction Resistance}

The plate constriction resistance mathematical model is based on the physical model presented in the schematic of Fig. 7. The derivation of the model is detailed in Mantelli and Yovanovich $^{8}$ and its expression is

$$
R_{\mathrm{ct}}=\frac{\bar{\psi}-\bar{\theta}}{q \pi\left(c^{2}-b^{2}\right)}
$$

where $\bar{\theta}$ is the mean temperature of the plate ring between the hole radius $a$ and the washer outer radius $b$, and $\bar{\psi}$ is the mean temperature of the plate ring between the washer outer radius and the plate outer radius $c$. The nondimensional form of Eq. (12) is

$$
R_{\mathrm{ct}}^{*}=\frac{\overline{\psi^{*}}-\overline{\theta^{*}}}{\pi\left(c^{*^{2}}-1\right)}
$$

where $c^{*}=c / b, \overline{\psi^{*}}$, and $\overline{\theta^{*}}$ are made nondimensional using Eq. (4). The expressions for $\overline{\psi^{*}}$ and $\overline{\theta^{*}}$ are very complex (several Bessel functions are included) and can be found in Mantelli and Yovanovich. ${ }^{8}$ If $\theta^{*}$ happens to be much smaller than $\psi^{*}$, then $\overline{\theta^{*}}$ can be eliminated, simplifying the equation considerably. Figure 8 presents the comparison of these terms. Because $\overline{\psi^{*}}$ and $\overline{\theta^{*}}$ depend on the same parameters, they were not varied independently in this study. Therefore, a parametric surface, generated by the variation of $a^{*}$ and $c^{*}$ over a wide

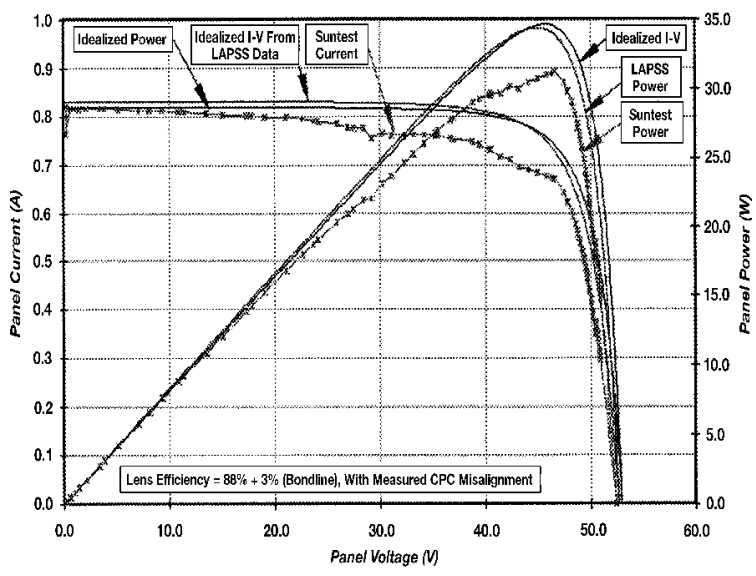

Fig. $8 \bar{\psi}^{*}$ and $\overline{\theta^{*}}$ for variable values of $\alpha *$ and $c^{*}$.

range of values, is used. This figure shows that the values of $\overline{\psi^{*}}$ are much larger than those of $\overline{\theta^{*}}$, at least twice as large. This means that $\theta^{*}$ can be removed from Eq. (13). Mantelli and Yovanovich ${ }^{8}$ showed that this simplification, in normal applications, causes a maximum error of around $2 \%$ in the overall resistance, for the cases where $a^{*}<0.8$ and $c^{*}<1 \underline{80}$.

Based on Eq. (13) and after the elimination of the $\overline{\theta^{*}}$ parameter, through some algebraic simplifications, Mantelli ${ }^{8}$ obtained this very simple equation for $R_{\mathrm{ct}}^{*}$

$$
R_{\mathrm{ct}}^{*}=\left(k^{*} / 2 \pi\right)\left(\ell_{n} c^{*}-\frac{3}{4}+\Phi\right)
$$

where $k^{*}$ and $\Phi$ are given by

$$
\begin{gathered}
k^{*}=\frac{k_{s} L_{s}}{k_{p} L_{p}} \\
\Phi=\frac{\left[K_{0}(\lambda) / K_{1}\left(\lambda a^{*}\right)\right]+\left[I_{0}(\lambda) / I_{1}\left(\lambda a^{*}\right)\right]}{\lambda\left\{\left[I_{1}(\lambda) / I_{1}\left(\lambda a^{*}\right)\right]-\left[K_{1}(\lambda) / K_{1}\left(\lambda a^{*}\right)\right]\right\}}
\end{gathered}
$$

where

$$
\lambda=\sqrt{\frac{h_{c w p}^{*} k_{s} b}{k_{p} L_{p}}}
$$

Mantelli and Yovanovich ${ }^{8}$ also showed that $\Phi$ can be considered to be equal to 0.1 for most of the practical applications.

In Fig. 9, the constriction resistance $R_{\mathrm{ct}}^{*}$ is compared to the washer path resistance $R_{w p a t h}^{*}$ and to the equivalent of these two resistances in series $R_{c w}^{*}$. From this plot, one observes that the constriction resistance is larger than the washer path resistance for most of the cases. Mantelli and Yovanovich ${ }^{9}$ showed that for low values of temperature, the contact resistances, present in the $R_{w \text { path }}^{*}$, can be the dominant term. Therefore, the washer path resistance should not be eliminated from the circuit.

\section{Radiation Resistance}

The analysis of the radiation path resistance between plates will not be presented here because its results are similar to those of the radiation resistance of the cell analysis. Therefore, this path is removed from the thermal circuit (see Fig. 2).

\section{Comparison Between the Complete and Simplified Circuit}

Figure 2 shows the resistance network before and after the application of cell concept and all of the simplifications proposed in this study. The complex initial circuit is now simplified to only three resistances in series. One implication of this simplification is the computational time required for the calculation of the overall thermal resistance. It is around 100 times greater for the complete network version, when an al- 


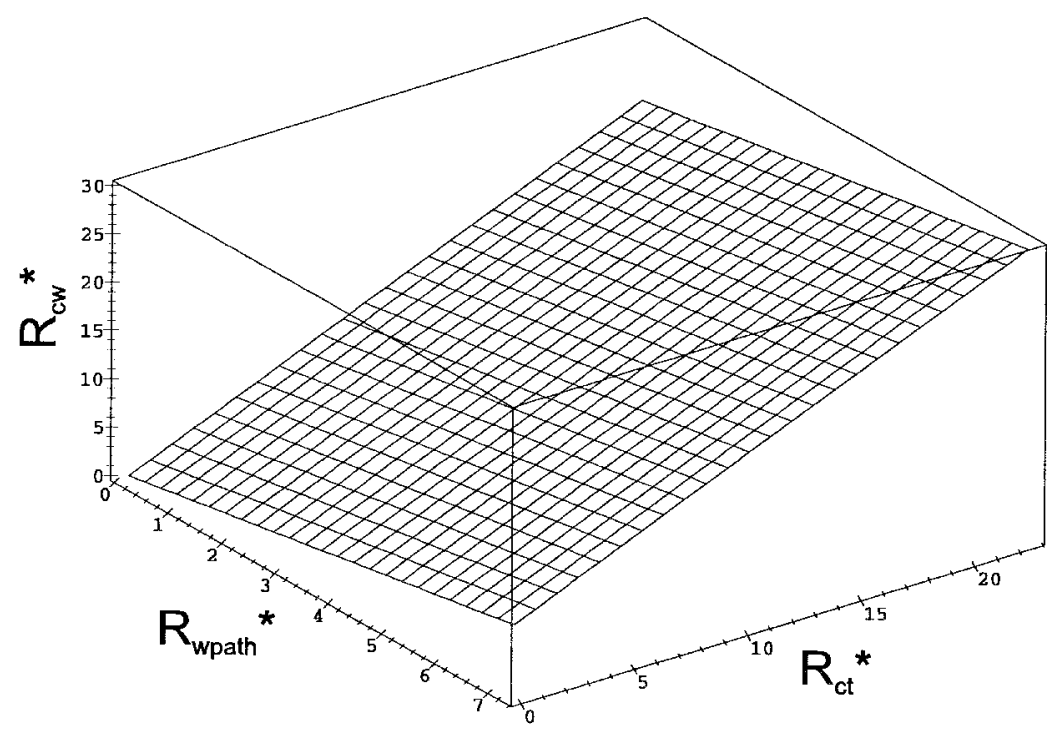

Fig. $9 R_{c w}^{*}$ as a function of $R_{w p a t h}^{*}$ and $R_{c}^{*}$.

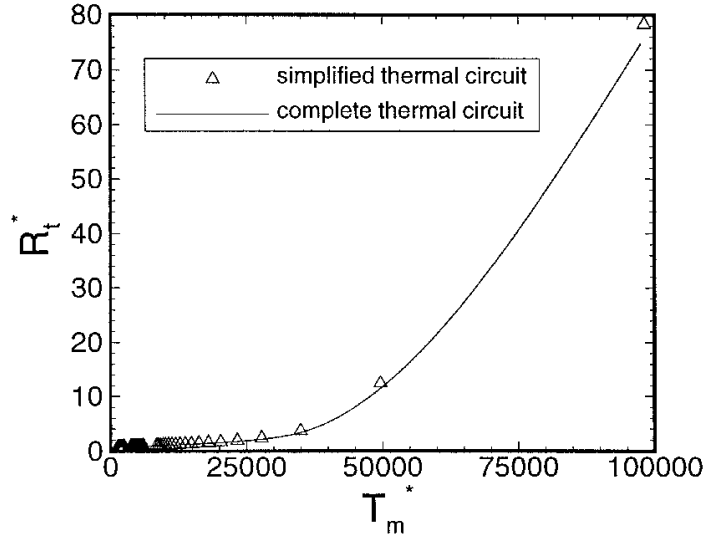

Fig. $10 R_{t}^{*}$ as a function of $T_{m}^{*}$ for the complete and simplified models.

gebraic manipulation software is used. The overall thermal resistance of bolted joints can be determined, using any hand calculator, from the following expression:

$$
R_{t}^{*}=2 R_{\mathrm{ct}}^{*}+R_{m w 1}^{*}+(n-1) R_{w w}^{*}, \quad n \geq 1
$$

where $R_{\mathrm{ct}}^{*}$ and $R_{w w}^{*}$ (cell resistance), are given by Eqs. (5), (7), and (14), and $R_{m w 1}^{*}$ is given by Eq. (5). $h_{m}^{*}$ is redefined as

$$
h_{m}^{*}=b k_{w} / k_{s} L_{w}
$$

Substituting Eqs. (5), (7), (14), and (18) in Eq. (17), one gets

$$
\begin{aligned}
R_{t}^{*} & =\left(k^{*} / \pi\right)\left(\ell n c^{*}-\frac{3}{4}+\Phi\right)+n\left[L_{s}^{*} / h_{m w 1}^{*} \pi\left(1-a^{* 2}\right)\right] \\
& +(n-1)\left[L_{s}^{*} / h_{c w w}^{*} \pi\left(1-a^{* 2}\right)\right]
\end{aligned}
$$

Figure 10 shows a plot of the comparison between the simplified and complete models applied to the mounting as described earlier. As $T_{m}$ is made nondimensional by $q$, and $q$ values increase faster than $T_{m}$, large values of $T_{m}^{*}$ means low $T_{m}$ temperatures. From this figure, one observes that $R_{t}^{*}$ increases with $T_{m}^{*}$, or in other words, the nondimensional resistance increases as the dimensional temperature decreases. The dependence of the resistance to the temperature is explained by the differential shrinkage of the aluminum and stainlesssteel materials. At low temperatures there is a reduction in the contact pressure and, therefore, an increase in the contact resistance. This effect is modeled at Mantelli et al. ${ }^{10}$ From Fig. 10 , one observes that the predictions of the overall thermal resistance, obtained through the simplified and the complete thermal circuits, are very close. The largest difference is $6 \%$ for very low temperature levels. This is very acceptable for most common applications, including satellites.

\section{Sensitivity Analysis}

Once the simplified circuit is obtained, a sensitivity analysis of the parameters used in the formulation of the overall thermal resistance of bolted joints is performed. The parameters that present the largest influence can be used to control the overall thermal resistance of bolted joints.

To perform the sensitivity analysis, the expression of the derivative of the total resistance relative to the parameter under consideration is obtained. It represents the sensitivity of the total resistance to this particular parameter. The range of variation adopted in this study is based on the nominal case. As a reference point, the position of the nominal case (present case) is shown in some plots.

The expression of the derivative of $R_{t}^{*}$ relative to $a^{*}$, which represents the sensitivity of $R_{t}^{*}$ to variations of $a^{*}$, is

$$
\frac{\partial R_{t}^{*}}{\partial a^{*}}=\frac{2 a^{*}}{\left(1-a^{* 2}\right)^{2}}\left[\frac{n L_{s}^{*}}{h_{m w}^{*} \pi}+\frac{(n-1) L_{s}^{*}}{h_{c}^{*} \pi}\right]
$$

On the other hand, the expression of the derivative of $R_{t}^{*}$ relative to $c^{*}$, which represents the sensitivity of $R_{t}^{*}$ to variations of $c^{*}$, is

$$
\frac{\partial R_{t}^{*}}{\partial c^{*}}=\frac{k^{*}}{\pi c^{*}}
$$

Observing Eq. (19), one may think that the first term (constriction resistance) is independent of the thicknesses of the plates and washers. This is not true, because the conductivities are inserted in the nondimensional parameters. For the study of the sensitivity of $R_{t}^{*}$ relative to $L_{s}^{*}, k^{*}$ is taken as a function of $L_{s}^{*}\left[k^{*}=\left(b k_{s} / k_{p} L_{p}\right) L_{s}^{*}\right]$. Then, $\partial R_{t}^{*} / \partial L_{s}^{*}$ is given by

$$
\begin{aligned}
\frac{\partial R_{t}^{*}}{\partial L_{s}^{*}} & =\frac{1}{\pi} \frac{b k_{s}}{k_{p} L_{p}}\left(\ell_{n} c^{*}-\frac{3}{4}+\Phi\right) \\
& +\frac{n}{h_{m w 1}^{*} \pi\left(1-a^{* 2}\right)}+\frac{n-1}{h_{c}^{*} \pi\left(1-a^{* 2}\right)}
\end{aligned}
$$


From this equation, one observes that $\partial R_{t}^{*} / \partial L_{s}^{*}$ is not dependent on $L_{s}^{*}$ and, for the present case study, $\partial R_{t}^{*} / \partial L_{s}^{*}=1.25$, demonstrating that $R_{t}^{*}$ is very sensitive to $L_{s}^{*}$. From Eq. (19) it is also observed that $R_{t}^{*}$ varies linearly with $L_{s}^{*}$.

The parameter $k^{*}$, defined as a ratio of conductivities and thicknesses, is not considered appropriated to study the influence of the conductivities of the materials in the overall thermal resistance. Instead of defining a new nondimensional parameter, the dimensional parameter $k_{s}$ was selected. After some algebraic manipulation, the dimensional resistance $R_{t}$ is obtained as a function of $k_{s}$. The dimensional form of the total resistance, in terms of $k_{s}$ is

$$
\begin{aligned}
R_{t} & =\frac{k_{w}}{\pi L_{p} k_{s}\left(k_{p}+k_{w}\right)}\left[\ln \left(\frac{c}{b}\right)-\frac{3}{4}+\Phi\right] \\
& +\frac{2 n L_{w} k_{p}}{\pi\left(b^{2}-a^{2}\right) k_{s}\left(k_{p}+k_{w}\right)}+\frac{(n-1) R_{q} / s}{1.25 k_{s}(P / H)^{0.95} \pi\left(b^{2}-a^{2}\right)}
\end{aligned}
$$

One should note that $k_{s}$ appears in the denominator of each term of this equation. Because $\Phi$ also depends on $k_{s}$ [see Eqs. (15) and (16)], the expression for $\partial R_{t} / \partial k_{s}$ is very complicated and will not be shown here.

Two different contact resistances are found in the present case, washer-washer and washer-plate. The expression for the sensitivity of $R_{t}^{*}$ with respect to $h_{c w w}^{*}$ is obtained from Eq. (19) and is given by

$$
\frac{\partial R_{t}^{*}}{\partial h_{c w w}^{*}}=\frac{-(n-1) L_{s}^{*}}{\pi\left(1-a^{* 2}\right) h_{c w w}^{* 2}}
$$

On the other hand, $h_{c w p}^{*}$ is one of the parameters of the argument of the Bessel functions used in the determination of the parameter $\Phi$ [see Eqs. (15) and (16)]. Therefore, the expression of $\partial R_{t}^{*} / \partial h_{c w p}^{*}$ is rather complicated and will not be shown here.

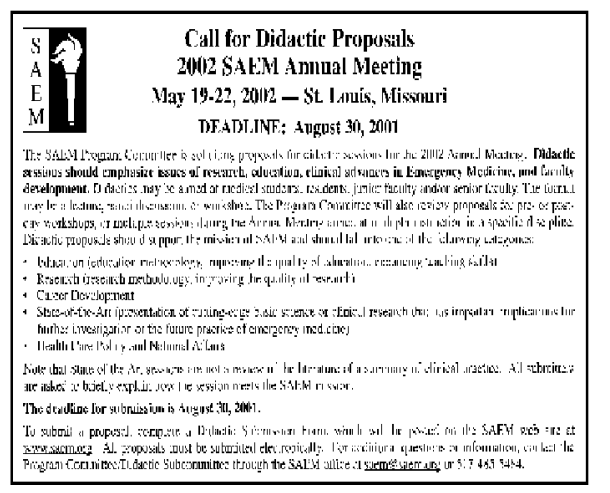

Fig. $11 \partial R_{t}^{*} / \partial a^{*}$ and of $R_{t}^{*}$ as a function of $a^{*}$.

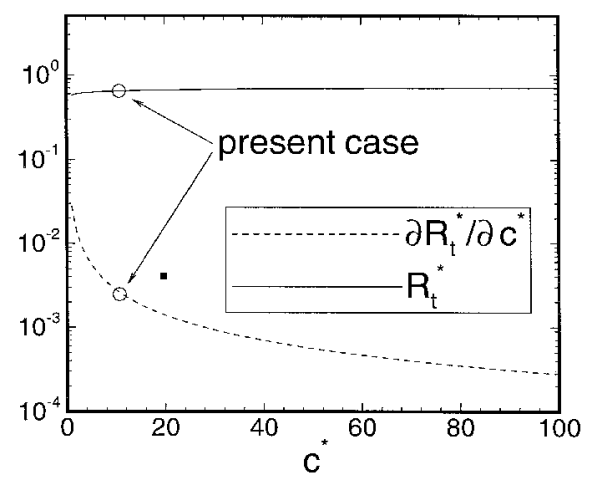

Fig. $12 \partial R_{t}^{*} / \partial c^{*}$ and of $R_{t}^{*}$ as a function of $c^{*}$.
In Fig. 11, a plot of the $\partial R_{t}^{*} / \partial a^{*}$ and of $R_{t}^{*}$ as a function of $a^{*}$ is presented. Observing this plot, one concludes that the overall thermal resistance is very sensitive to the $a^{*}$ parameter, for $a^{*} \geq 0.8$. For $0<a^{*}<0.8$, the overall thermal resistance is almost constant. Actually, as $a^{*}$ tends to 1 , the heat transfer area tends to zero and, therefore, the material and contact resistances tend to infinity.

Figure 12 presents a plot of the $\partial R_{t}^{*} / \partial c^{*}$ and of $R_{t}^{*}$ as a function of $c^{*}$. A wide range of variation was selected for the $x$ axis (the present case points are close to the left side of the graph). This plot shows that the overall thermal resistances are neither very sensitive nor very dependent of the $c^{*}$ parameter. The sensitivity increases as $c^{*}$ tends to 1 , its smallest possible value. On the other hand, as the value of $c^{*}$ increases, the sensitivity decreases and the overall thermal resistance increases slightly. Actually, this is an interesting result for practical applications. When several bolted joints are used in parallel for the fixation of an electronic box in a satellite panel, for instance, the question whether one junction thermally affects the other always arises. For $c^{*} \gtrsim 10$, this parameter has so little influence on $R_{t}^{*}$ that this region can be considered not to be affected by the bolted joint. Note that $b$ is the washer o.d. (or the radio of the contact area, if there are no washers between plates). If the neighboring bolted joint is within this region $\left(c^{*}<10\right)$, they are considered thermally dependent. Otherwise, they are independent and their resistances can be considered in parallel.

Figure 13 presents a plot of the dimensional $R_{t}$ and $\partial R_{t} / \partial k_{s}$ as a function of $k_{s}$, from which one observes that the total resistance is very sensitive and dependent on the parameter $k_{s}$, for low values of $k_{s}$. The negative values of the sensitivity curve show that when increasing $k_{s}$ the $R_{t}$ then decreases. This result was expected, because the thermal resistance is inversely proportional to the conductivity of the materials.

Similar conclusions can be obtained from the study of the influence of the contact conductances on $R_{t}^{*}$. Only the plots of $\partial R_{t}^{*} / \partial h_{c w w}^{*}$ and $R_{t}^{*}$ against $h_{c w w}^{*}$ are presented (Fig. 14), because they are very similar to the plots of $\partial R_{t}^{*} / \partial h_{c w p}^{*}$ and $R_{t}^{*}$ against $h_{c w p}^{*}$, although they represent the influence of different contacts. They are also similar to the curves of Fig. 13 and of the projections of the Fig. 5 surface over the $x z$ and $y z$ planes. From Fig. 14 it can be observed that for low values of the nondimensional contact conductances the overall resistance increases quickly. This observation agrees with the conclusions of the Analysis of the Cell Resistance section in this paper. These high resistances can be a consequence of the loss of the physical contact between the surfaces. The zone where this instability occurs is referred as instability zone. On the other hand, the overall thermal resistance is almost not affected for nondimensional conductances larger than 2.3. For many joints the contact resistance may not be the most important parameter as it has been considered in many works found in the literature. For the joint described in the Bolted Joint Configuration section in this

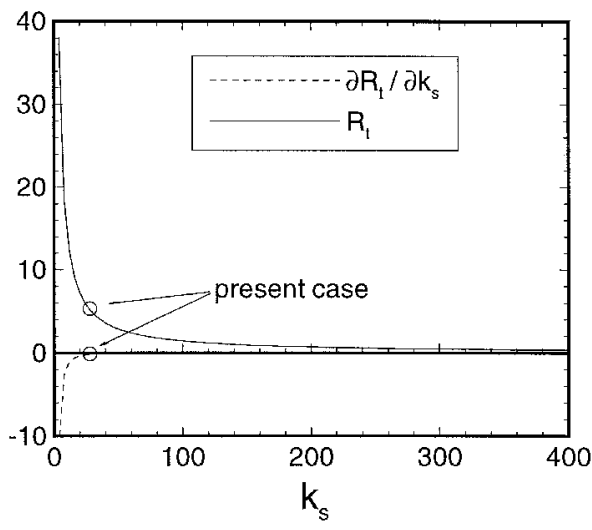

Fig. $13 \partial R_{t} / \partial k_{s}$ and of $R_{t}$ as a function of $k_{s}$. 


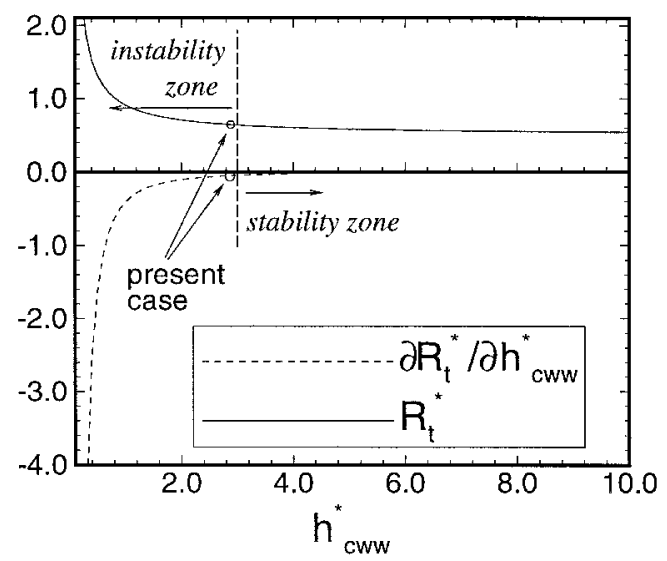

Fig. $14 \partial R_{t}^{*} / \partial h_{c w w}^{*}$ and of $R_{t}^{*}$ as a function of $h_{c w w}^{*}$

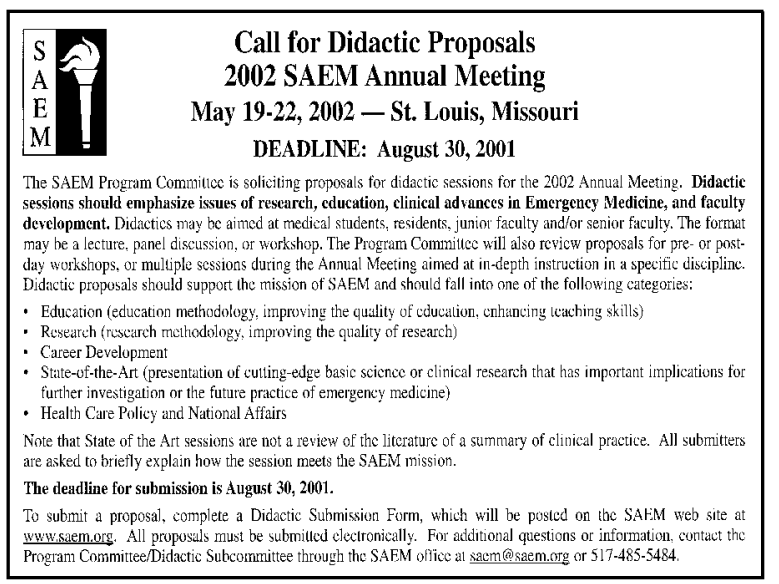

Fig. 15 Comparison between Mantelli and Yovanovich ${ }^{8}$ data and model.

paper, all of the contact resistances represent $11 \%$ of the total resistance.

\section{Comparison Between Model and Literature Data}

Refer to Mantelli and Yovanovich ${ }^{13}$ for a comparison between the present model and the extensive parametric experimental work in bolted joints shown in Mantelli and Yovanovich. ${ }^{14}$ In the present study one set of experimental data obtained for a mounting tested by Mantelli and Yovanovich ${ }^{15}$ was selected. The geometric and physical parameters of the experimental mounting are those presented earlier, except for $n$, which is taken as 1 . The tested washers were flat. Therefore, the effect of the curvature was not considered in the model or the experiment. The comparison between the model [Eq. (19)], and data is shown through the plot of $R_{t}^{*}$ against $T_{m}^{*}$, in Fig. 15 (remember that $T_{m}^{*}$ decreases as $T_{m}$ increases). The vertical bars in this plot represent the experimental errors. As one observes, the mean difference between model and data is less than $13 \%$. This comparison is very good, especially considering the complexity of the bolted joint. The theoretical overall resistances are expected to underpredict the experimental results, as they do, because of several simplifications adopted in the model (no constriction in bolts, washers, nuts, uniform contact pressure, etc.). $P_{i}$ in Fig. 15 is the assembling contact pressure. This pressure was measured only during the assembling procedure. For the experimental conditions, the actual contact pressure is estimated through thermal stress analytical models. 9,10

The authors are not aware of any other experimental data of bolted joints with washers between plates. In the attempt to compare the present model with other models and data from the literature, the "no washer between plate case" is used. The

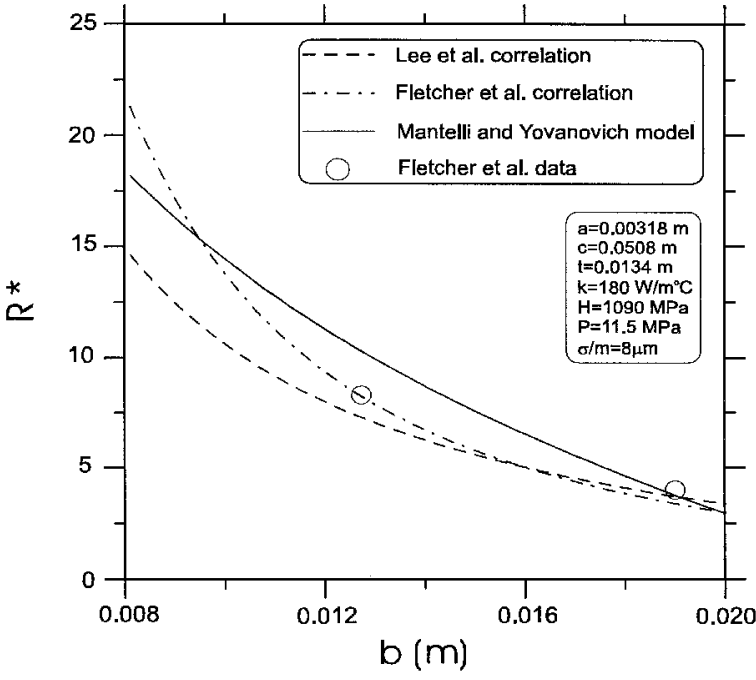

Fig. 16 Comparison between the present model and correlations and data from literature.

contact area, which is given by the washer dimensions for the case of "washers between plates," is estimated using the Song et al. ${ }^{16}$ model. Figure 16 shows the comparison among the Lee et al. ${ }^{7}$ correlation, the Fletcher et al. ${ }^{6}$ correlation, and experimental data, and the present model. In this plot, $R^{*}$ is defined as $R^{*}=\left(R_{\text {total }}-R_{\text {bulk }}\right) / R_{\text {bulk, }}$, where $R_{\text {total }}$ is the total resistance including the constriction resistance, and $R_{\text {bulk }}$ is the resistance without the constriction $\left(R_{\text {bulk }}=L / k A\right)$, according to the Fletcher et al. ${ }^{6}$ definition. The parameters used in this comparison are shown in this figure. One observes that the comparison among all of these models and data is quite good. Fletcher et al. simulated the constriction resistance of plates in bolted joints using an electrolytic tank. As no contact resistance was included, the present model is expected to overpredict the Fletcher et al. experimental data, as it does.

\section{Conclusions}

From the extensive work presented in this paper, many conclusions were obtained. The most important are summarized here.

1) All of the radiation resistances can be removed from the thermal circuit with negligible error.

2) The $R_{\text {cell }}^{*}$ presents a weak dependence of $h_{c}^{*}$, for $h_{c}^{*}>3$.

3) The $h_{c}^{*}$ and $h_{m}^{*}$ parameters influence $R_{\text {cell }}^{*}$ similarly.

4) For most applications, the bolt heat transfer path can be removed from the thermal circuit.

5) $\bar{\theta}^{*}$ (inner ring temperature) can be removed from the constriction resistance equation with errors inferior to $2 \%$, for $a^{*}<0.8$ and $c^{*}>180$.

6) The simplified circuit, which consists of three resistances in series, compares with the complete one within $6 \%$, for the case analyzed in the present work. This difference is expected to be approximately the same for other cases.

7) The total resistance is very sensitive to $a^{*}$, for $a^{*}>0.8$. For $a^{*}<0.8$, the precision with which this parameter is determined is not relevant.

8) The total resistance is not very dependent nor very sensitive to variations of $c^{*}$, if $c^{*}>10$.

9) $L_{s}^{*}$ is a very important parameter for the calculation of $R_{t}^{*}$ and should be determined accurately.

10) $n$ is an important parameter.

11) $R_{t}$ is very influenced and sensitive to variations of $k_{s}$, particularly for small values of $k_{s}$, which should be accurately determined.

12) Similar observations can be made for the influence of the parameters $h_{c w w}^{*}$ and $h_{c w p}^{*}$.

13) $R_{t}^{*}$ is almost independent of $h_{c w w}^{*}$ and $h_{c w p}^{*}$, for $h_{c w w}^{*}$ and $h_{c w p}^{*}>3$. This remark is similar to the conclusion 2 in this 
section. This is an interesting observation because many works dealing with thermal conductance of bolted joints are about the contact conductance, which may not be the most important parameter.

Also, from this work, a thermal engineer can determine, for other bolted joint configurations, whether the bolt and/or radiation paths should not be considered as thermal resistance paths in his/her model.

The comparison between the present model and experimental data shows that the model is adequate for the determination of the overall thermal resistance. The comparison of this model with literature model and data is very good, which demonstrates that the thermal circuit composed of three resistances in series should be used because of its simplicity, precision, and accuracy.

\section{Acknowledgments}

M. B. H. Mantelli acknowledges the financial support from CNPq (Brazilian Research Agency) under Grant 202021891.1, and M. M. Yovanovich acknowledges the support of the Natural Sciences and Engineering Research Council.

\section{References}

'Aron, W., and Colombo, G., "Controlling Factors of Thermal Resistance Across Bolted Joints in a Vacuum Environment," American Society of Mechanical Engineers, Paper 63-WA-196, Nov. 1963.

Elliot, D. H., "Thermal Conduction Across Aluminum Bolted Joints," American Society of Mechanical Engineers, Paper 65-HT-53, Aug. 1965.

Fontenot Jr., J. E., "The Thermal Conductance of Bolted Joints," Ph.D. Dissertation, Louisiana State Univ. and Agricultural and Mechanical College, Baton Rouge, LA, Jan. 1968.

${ }^{4}$ Mittlebach, M., Vogd, L. S., Fletcher, L. S., and Peterson, G. P., "The Interfacial Pressure Distribution and Thermal Conductance of Bolted Joints," American Society of Mechanical Engineers, HTD, Vol. 212, New York, 1992, pp. 9-17.

SMaddren, J., "Thermal Contact Resistance of Bolted Joints at Cryogenic Temperature," Ph.D. Dissertation, Univ. of California, Santa Barbara, CA, Feb. 1994.
Fletcher, L. S., Peterson, G. P., Madhusudana, C. V., and Groll, E., "Constriction Resistance Through Bolted and Riveted Joints," Journal of Heat Transfer, Vol. 112, Nov. 1990, pp 857-863.

${ }^{7}$ Lee, S., Yovanovich, M. M., and Moran, K. P., "Analysis of Thermal Constriction Resistance in Bolted Joints," International Journal of Microcircuits and Electronic Packaging, Vol. 16, No. 2, 1993, pp. $125-136$.

¿Mantelli, M. B. H., and Yovanovich, M. M., "Compact Analytical Model for Overall Thermal Resistance of Bolted Joints," International Journal of Heat and Mass Transfer, Vol. 41, No. 10, 1998, pp. 12251266.

'Mantelli, M. B. H., and Yovanovich M. M., “Analytical Model for the Overall Thermal Resistance of Bolted Joints," AIAA Paper 95-0420, Jan. 1994.

${ }^{10}$ Mantelli, M. B. H., Sridhar, M. R., and Yovanovich, M. M., "Influence of the Elastic and Plastic Contact Models on the Overall Thermal Resistance of Bolted Joints," 11th Annual IEEE Semiconductor Thermal Measurement and Management, San Jose, CA, Feb. 1995.

${ }^{11} Y o v a n o v i c h$, M. M., "Thermal Contact Correlations," AIAA Paper 81-1161, June 1981

${ }^{12}$ Cooper, M. G., Mikic, B. B., and Yovanovich, M. M., "Thermal Contact Conductance," International Journal of Heat and Mass Transfer, Vol. 12, No. 2, 1969, pp. 279-300.

${ }^{13}$ Mantelli, M. B. H., and Yovanovich, M. M., "Overall Thermal Resistance of Satellite Bolted Joints: Comparison of Analytical Model with Experimental Data," AIAA Paper 96-0240, Jan. 1996.

${ }^{14}$ Mantelli, M. B. H., and Yovanovich, M. M., "Experimental Determination of the Overall Thermal Resistance of Bolted Joints," Journal of Thermophysics and Heat Transfer, Vol. 10, No. 1, 1996, pp. $177-179$.

${ }^{15}$ Mantelli, M. B. H., and Yovanovich, M. M., "Experimental Setup for Measurement of Overall Thermal Resistance of Bolted Joints," Proceedings of the 6th Brazilian Congress of Engineering and Thermal Sciences (Florianópolis, SC, Brazil), BCM, São Paulo, Brazil, 1996, pp. 797-802.

${ }^{16}$ Song, S., Park, C., Moran, K. P., and Lee, S., "Contact Area of Bolted Joint Interface: Analytical, Finite Element Modelling and Experimental Study," Computer Aided Design in Electronic Packaging, Vol. 3, American Society of Mechanical Engineers, New York, 1992, pp. $73-81$. 